This is the author's final, peer-reviewed manuscript as accepted for publication. The publisher-formatted version may be available through the publisher's web site or your institution's library.

\title{
Influence of allowable interruption period on estimates of accelerometer wear time and sedentary time in older adults
}

Emily L. Mailey, Neha P. Gothe, Thomas R. Wójcicki, Amanda N. Szabo, Erin A. Olson, Sean P. Mullen, Jason T. Fanning, Robert W. Motl, and Edward McAuley

\section{How to cite this manuscript}

If you make reference to this version of the manuscript, use the following information:

Mailey, E. L., Gothe, N. P., Wójcicki, T. R., Szabo, A. N., Olson, E. A., Mullen, S. P., ... McAuley, E. (2014). Influence of allowable interruption period on estimates of accelerometer wear time and sedentary time in older adults. Retrieved from http://krex.ksu.edu

\section{Published Version Information}

Citation: Mailey, E. L., Gothe, N. P., Wójcicki, T. R., Szabo, A. N., Olson, E. A., Mullen, S. P., ... McAuley, E. (2014). Influence of allowable interruption period on estimates of accelerometer wear time and sedentary time in older adults. Journal of Aging and Physical Activity, 22(2), 255-260.

Copyright: @ 2014 Human Kinetics, Inc.

Digital Object Identifier (DOI): doi:10.1123/JAPA.2013-0021

Publisher's Link: http://dx.doi.org/10.1123/JAPA.2013-0021

This item was retrieved from the K-State Research Exchange (K-REx), the institutional repository of Kansas State University. K-REx is available at http://krex.ksu.edu 
Running head: Accelerometer wear time in older adults

Influence of allowable interruption period on estimates of accelerometer wear time and sedentary time in older adults

\author{
Emily L. Mailey ${ }^{1 \mathrm{a}}$, Neha P. Gothe², Thomas R. Wójcicki ${ }^{2}$, Amanda N. Szabo², Erin A. Olson², \\ Sean P. Mullen ${ }^{2}$, Jason T. Fanning ${ }^{2}$, Robert W. Motl' ${ }^{2}$, \& Edward McAuley ${ }^{2}$ \\ ${ }^{1}$ Department of Kinesiology, Kansas State University \\ ${ }^{2}$ Department of Kinesiology \& Community Health, University of Illinois at Urbana-Champaign \\ ${ }^{a}$ Note: Research was completed at University of Illinois at Urbana-Champaign
}

\title{
Corresponding Author:
}

Emily L. Mailey, PhD

Kansas State University

Department of Kinesiology

8D Natatorium

Manhattan, KS 66506

Phone: (785) 532-7287

Fax: (785) 532-6486

Email: emailey@ksu.edu 


\begin{abstract}
The criteria one uses to reduce accelerometer data can profoundly influence the interpretation of research outcomes. The purpose of this study was to examine the influence of three different interruption periods (i.e., 20, 30, and 60 minutes) on the amount of data retained for analyses and estimates of sedentary time among older adults. Older adults $\left(N=311 ; M_{\text {age }}=71.1\right)$ wore an accelerometer for seven days and reported wear time on an accelerometer log. Accelerometer data were downloaded and scored using 20, 30, and 60-minute interruption periods. Estimates of wear time derived using each interruption period were compared to self-reported wear time, and descriptive statistics were used to compare estimates of sedentary time. Results showed a longer interruption period (i.e., 60 minutes) yields the largest sample size and the closest approximation of self-reported wear time. A short interruption period (i.e., 20 minutes) is likely to underestimate sedentary time among older adults.
\end{abstract}




\section{Introduction}

Increasing physical activity and reducing inactivity among older adults is an important public health priority. In order to understand physical activity patterns and assess the effectiveness of interventions, it is important to be able to accurately measure physical activity (Dishman, Washburn, \& Schoeller, 2001). Self-report physical activity questionnaires are widely used by researchers; however accelerometry is becoming an increasingly popular method to collect objective unbiased physical activity data (Troiano, et al., 2008). Accelerometers are electronic motion sensors that measure the quantity and intensity of movement and provide detailed, objective physical activity data without placing a great deal of burden on participants (Esliger, et al., 2005). In older adults, accelerometers might help overcome several of the limitations of physical activity questionnaires (Shephard, 2003). For example, they reduce recall and/or self-report bias, and they capture light, brief activity, which is characteristic of older adults, but may not be included in participants’ own cognitive appraisals of their activity.

On the other hand, accelerometers produce enormous amounts of raw data that must be adequately processed in order to analyze and interpret them (Ward, et al., 2005). When assessing free-living physical activity or inactivity, the researcher's goal is to include data that reflect actual wear time and eliminate data that reflect non-wear time (i.e., monitor is removed for bathing, sleeping, etc.). Because wear time is different for each day and each participant, participants are often asked to complete logs indicating when they wore the monitor each day (Esliger et al., 2005). Using such logs to differentiate wear time from non-wear time on a day-today basis may not be feasible, however, when working with many participants. Thus, for large studies, it is preferable to use automated algorithms to include/exclude data based on pre- 
determined criteria (Masse, et al., 2005; Winkler, et al., 2012). For example, the current consensus in the physical activity literature is that ten hours of daily wear is long enough to eliminate days when the monitor was not worn for a significant proportion of waking hours, but low enough to preserve days with sufficient data (Troiano, et al., 2008). Increasing this figure further could lead to substantial reductions in sample size and statistical power (Colley, Gorber, \& Tremblay, 2010).

The issue of estimating wear time becomes more complex when one considers that inactivity is part of human behavior so it is not possible to simply eliminate zero counts from the data, as these may represent sedentary time, as opposed to non-wear time (Evenson \& Terry, 2009). Instead, researchers must identify an allowable interruption period in which a certain number of consecutive zeros within an hour is acceptable (Colley, et al, 2010). Modifying this interruption period can drastically alter the number of valid hours and, in turn, the amount of data retained for analyses. A shorter interruption period could potentially eliminate useful data by incorrectly classifying inactivity as non-wear time, and thus inflate the percentage of time spent in light, moderate, and vigorous activity (Masse, et al., 2005). Alternatively, although a longer interruption period is less likely to misclassify sedentary time as non-wear time (Choi, Liu, Matthews, \& Buchowski, 2011), it could erroneously include non-wear time in the final analysis, thus potentially diluting physical activity outcomes. Importantly, since zero counts reflect either non-wear or inactivity, sedentary time assessments will be directly affected by altering the interruption period. Given the recent interest in reducing sedentary time as a valuable behavioral outcome (Owen, Healy, Matthews, \& Dunstan, 2010), deriving accurate estimates of sedentary time using accelerometers is an important research objective. 
Although the interruption period can significantly influence accelerometer data, and thus research outcomes, the criteria used during accelerometer data reduction are rarely reported. In their review of studies published in 2003-2004, Masse et al. (2005) found only 12.5\% reported the allowable interruption period. The most commonly used period was 10 minutes of consecutive zeros (e.g., Brage, et al., 2004; Riddoch et al., 2004), but a majority of these studies were conducted with children, who exhibit activity patterns that are drastically different from adults. For example, a controlled experiment of monitored sedentary time compared 10, 20, 30, and 60-minute interruption periods and demonstrated that wear time algorithms were much less likely to misclassify inactivity as monitor removal in children than adults, for whom the 60minute period was deemed optimal (Rowlands, et al., 2010). Similarly, Evenson and Terry (2009) compared 20,40, and 60-minute interruption periods in a sample of postpartum women and concluded that the 60-minute period best reflected actual wear time, however their participants did not complete accelerometer logs so their conclusion was based on the assumption that the women followed the study protocol. These studies recognize that identifying wear/non-wear time is an important step in accelerometer data reduction, but no studies have addressed this question among older adults, for whom activity patterns might be affected by different lifestyles, chronic disease states, and functional limitations.

The purpose of the present study was to examine the influence of three different interruption periods (i.e., 20, 30, and 60 minutes) on the amount of data retained for analyses and estimates of sedentary time among older adults. We compared estimates of wear time derived using each of the interruption periods to self-reported wear time reported on participant logs to determine the relative accuracy of each algorithm. This study aimed to provide another step towards the goal of standardizing accelerometer data reduction procedures within specific 
populations such as older adults, which would allow for consistent data processing, and thus enhance the comparability of results between studies.

\section{Methods}

In the present study we report analysis of the baseline accelerometer data from a large 6month randomized controlled exercise trial. Complete details of the study design, methods, and primary outcomes have been reported elsewhere (McAuley, et al., 2012; McAuley, et al., 2013). Participants

Participants were older adults recruited to participate in a home-based exercise intervention. To be included in the study, participants had to be at least 65 years old, report being inactive during the previous six months, and have no medical conditions exacerbated by physical activity participation, as indicated by physician authorization. Following initial contact by telephone, participants completed a pre-screening interview to determine whether they met inclusion criteria and consented to have their physician contacted. Participants were excluded from participation if they did not meet the above criteria or their physician refused to provide approval for participation.

Procedures

All procedures were approved by the University Institutional Review Board. Participants who met all inclusionary criteria signed an informed consent document and then were mailed an accelerometer and a log with instructions. They were instructed to wear the accelerometer during all waking hours, except when bathing or swimming, for seven consecutive days. In addition, participants were asked to record the exact times they started and stopped wearing the monitor each day, including periods of monitor removal, on an accelerometer log. The packet included 
detailed written instructions for wearing the accelerometer and completing the log, an index of answers to frequently asked questions, and a telephone number they could call if they had any questions. Following the 7-day wear period, participants returned the accelerometer and log.

Measures

Accelerometer

The Actigraph accelerometer (Actigraph, Pensacola, FL; Model GT1M or GT3X) was used for the present study. Previous validation studies have demonstrated strong agreement between activity counts collected from the GT1M and GT3X, suggesting that these models can be used interchangeably for vertical axis data (Sasaki , John, \& Freedson, 2011). Both models are lightweight and enclosed in an identical plain red case that does not provide any physical activity feedback to participants while they are wearing it. Once downloaded, data collected from the accelerometer are displayed as activity counts, which reflect raw accelerations that have been digitized, converted, and summed over a specified epoch length (e.g., 1 minute). The activity counts are directly related to physical activity intensity. For the current study, the accelerometer was secured on an elastic belt and participants were instructed to wear the monitor on their nondominant hip.

Accelerometer data were scored by two investigators using MeterPlus version 4.2 (Santech, Inc.; San Diego, CA). We were interested in defining non-wear time using 20, 30, and 60-minute interruption periods, as these have been frequently used in data reduction previously (Masse, et al., 2005). To score the data, the investigators consulted the participants' activity logs and saved the seven days on which the participant indicated he or she wore the monitor. The raw data were scanned for anomalies (e.g., strings of consecutive identical non-zero numbers, counts exceeding the threshold for human movement) and excluded in such cases. Based on the 
consensus in the existing literature, a minimum of ten valid hours of wear time were required for a day to be considered valid. Although the number of valid hours (and thus the number of valid days) changed when the interruption period increased from 20 to 30 to 60 minutes, the same seven days were saved for all three periods for each participant. This resulted in three separate batches of files. An established cut-point $(<100)$ was used to estimate minutes of sedentary time (Freedson, Melanson, \& Sirard, 1998), which were subsequently converted to daily hours of sedentary time (minutes/60). In addition, we calculated the percentage of total wear time spent in sedentary activity by dividing the number of minutes of sedentary time (based on each interruption period) by the total self-reported minutes of wear time.

Activity $\log$

Participants recorded the exact times they wore the accelerometer each day on an activity log, which was used as a criterion measure of wear time. The exact number of hours of wear per day was calculated by summing the hours of reported wear time and subtracting any time the participant reported he or she had removed the monitor (e.g., bathing). The number of valid days was calculated by summing the number of days the participant reported wearing the accelerometer for at least ten hours.

Data analysis

All subsequent analyses were conducted using Predictive Analytics Software (PASW, version 18). First, we were interested in the extent to which changes in the interruption period would affect the amount of data retained. To examine this, we calculated the average number of valid hours per day for each of the three interruption periods and used t-tests and correlation analyses to compare these values to self-reported wear time. Additionally, we calculated the 
percentage of participants with one, two, three, four, five, six, and seven days of valid data when the interruption period was increased from 20 to 30 to 60 minutes and compared these percentages to those derived using self-reported wear time.

Next, we examined the influence of interruption period on estimates of sedentary time. Descriptive statistics were used to compare estimates of sedentary time based on a 20, 30, and 60 minute interruption period. For all analyses, we used non-parametric tests (i.e., Wilcoxon signed ranks tests and Spearman's correlations) in the event that data were skewed.

\section{Results}

Participant characteristics

A total of 311 individuals enrolled in the study and wore the accelerometer. The mean age of all participants was $71.12(S D=5.13)$ years, and the majority were female (76.7\%), welleducated (43.3\% with at least a college degree), and relatively affluent (53.3\% with an annual income $>\$ 40,000)$. Of the 311 participants, 298 (95.8\%) submitted an activity log.

Effect of interruption period on estimates of wear time

The mean wear time (hours per day) was calculated based on the activity logs and the three different allowable interruption periods. Figure 1 displays the mean values. On average, participants reported wearing the accelerometer for $13.83(S D=1.67)$ hours per day. When the data were scored using MeterPlus, participants had 10.32 valid hours $(S D=2.39)$ with a 20minute interruption period, 12.48 valid hours $(S D=2.16)$ with a 30-minute interruption period, and 14.96 valid hours $(S D=2.04)$ with a 60 -minute interruption period. Wilcoxon signed ranks tests revealed the self-reported mean hours per day were significantly higher than the mean hours calculated using a 20-minute interruption period $Z=14.89, p<.001, r=.86$ and a 30-minute interruption period $Z=13.38, p<.001, r=.78$, and significantly lower than the mean hours 
calculated using a 60-minute interruption period $Z=13.86, p<.001, r=.80$. Correlations between self-reported hours of wear and estimated valid hours of wear were .51, .71, and .87 (all ps<.001) for 20, 30, and 60-minute interruption periods, respectively. Comparison of these correlations using Fisher's r-to-z transformation revealed that all three correlations were significantly different from each other.

Table 1 and Figure 2 display the percentage of participants with a minimum of 1-7 valid days (i.e., minimum of 10 valid hours) when the interruption period increased from 20 to 30 to 60 minutes, as well as the number of participants with 1-7 days of self-reported wear based on the logs. For each day, the number of participants with at least 10 valid hours increased as the duration of the interruption period increased, although the difference between 20 and 30 minutes was more dramatic than the difference between 30 and 60 minutes. The data reflecting selfreported wear time most closely matched the wear time data derived using a 60-minute interruption period. Correlations between self-reported days of wear and estimated valid days of wear were .33, .55, and .56 (all ps<.001) for 20, 30, and 60-minute interruption periods, respectively. Analyses using Fisher’s r-to-z transformation revealed that 20 minutes was significantly different from 30 and 60 minutes, but 30 and 60 minutes did not differ from each other.

Effect of interruption period on estimates of sedentary time

Next we examined the influence of the allowable interruption period on sedentary time, expressed as average daily hours of sedentary time, and percentage of total wear time across the week. Daily hours of sedentary time increased from $8.55(S D=1.43)$ hours to $8.93(S D=1.43)$ hours to 9.74 ( $S D=1.62$ ) hours for 20 , 30, and 60-minute interruption periods, respectively. Similarly, the proportion of total wear time spent in sedentary activity increased from $40.0 \%$ 
( $S D=.196)$ to $57.7 \%(S D=.127)$ to $68.8 \%(S D=.107)$. Wilcoxon signed ranks tests revealed significant differences among all estimates of sedentary time (all $p$ 's $<.001$ ).

\section{Discussion}

Accelerometers can provide relatively accurate, reliable physical activity data and appear to be a promising means for measuring physical activity and inactivity in older adults. In order to maximize the quality of the data they provide, however, it is critical to establish standard procedures for accelerometer data reduction that are supported by empirical evidence. The length of the allowable interruption period can drastically impact the quantity and quality of data retained for analyses, but has received minimal attention to date in older adult populations. The purpose of this study was to examine the influence of three different interruption periods on the amount of data retained for analyses and estimates of sedentary time among older adults.

As expected, increasing the interruption period had a significant impact on sample size. Specifically, when using a 60-minute period, the greatest number of participants had at least 10 valid hours of data. When the interruption period was changed to 30 minutes, this percentage dropped slightly, and when further restricting the interruption period to 20 minutes, there was a more drastic reduction in the number of participants with valid data. For example, if one was interested in only retaining participants with at least five valid days, as recommended previously by Troiano et al. (2008), the percentage of data retained would be $59.2 \%, 89.4 \%$, and $97.7 \%$ for 20, 30, and 60 minute interruption periods, respectively. These results suggest that whereas an interruption period of 30 minutes may be conservative enough to retain a majority of participants, further reducing this period to 20 minutes may severely compromise sample size. It is likely that for older adults, who may have substantial bouts of sedentary activity throughout 
the day, an interruption period of 20 minutes is too restrictive and would incorrectly classify inactivity as non-wearing time.

Comparison between self-reported wear time and wear time estimated using each of the interruption periods substantiated these findings and further supported the use of a longer interruption period. Specifically, estimated days of wear derived using a 60-minute interruption period were most strongly correlated with self-reported valid days of wear. Thus, extending the interruption period appears to not only increase the available sample size, but also more closely reflect actual wear time.

Changes in the interruption period also significantly affected estimates of sedentary time, particularly when sedentary time was expressed as a percentage of total wear time. Although we did not have a criterion measure of sedentary time with which to compare the estimates, population-based studies have estimated older adults spend over $60 \%$ of their waking hours engaged in sedentary activities (Matthews, et al., 2008). In addition, because participants who reported engaging in physical activity more than two days per week were excluded from this study, the proportion of sedentary time should have been relatively high. Thus, the estimate of 40.0\% derived using a 20 -minute interruption period is likely a severe underestimation of sedentary time.

There are a number of strengths and limitations to this study. To our knowledge this is the first study to have investigated the issue of allowable interruption period in a large sample of older adults. Although the sample was relatively homogenous in that a majority of participants were white, highly educated, fairly affluent, and relatively sedentary, we believe these findings make a novel contribution to the study of accelerometer data collection and reduction, and bring 
us a step closer to the goal of establishing standardized procedures for data reduction. Although our findings suggest a 60-minute interruption period is likely to be optimal within this population, these findings need to be replicated in other samples of older adults. We would caution future researchers to avoid generalizing these findings to other populations (e.g., children/young adults, individuals with chronic diseases that affect physical and/or cognitive function), as activity patterns in these groups may differ markedly from those exhibited by older adults. This study did not utilize a criterion measure of sedentary time, so our results relative to this outcome are purely descriptive and should be compared to alternative measures of sedentary time in future studies to ensure algorithms accurately distinguish it from non-wear time. Future studies might also consider improving data reduction algorithms by removing spurious data (i.e., 1-2 nonzero counts surrounded by long strings of zero counts; Evenson \& Terry, 2009; Winkler, et al., 2012).

In conclusion, our findings indicate a longer interruption period (i.e., 60 minutes) yields the largest sample size and the closest approximation of self-reported wear time. A short interruption period (i.e., 20 minutes) is likely to underestimate sedentary time among older adults. These results suggest researchers should use a longer interruption period when collecting accelerometer data from older adults in order to maximize sample size and provide the most accurate estimation of wear time and sedentary time.

\section{Acknowledgements}

The authors gratefully acknowledge Ms. Susan Herrel for her work as project coordinator. This work was supported by the National Institute on Aging (2 R01AG020118 - 05). 


\section{References}

Brage, S., Wareham, N., Wedderkopp, N., Andersen, L., Ekelund, U., Froberg, K., et al. (2004). Features of the metabolic syndrome are associated with objectively measured physical activity and fitness in Danish children: The European Youth Heart Study. Diabetes Care, 27, 2141-2148.

Choi, L., Liu, Z., Matthews, C. E., \& Buchowski, M. S. (2011). Validation of accelerometer wear and nonwear time classification algorithm. Medicine \& Science in Sports \& Exercise, 43, 357-364. doi: 10.1249/MSS.0b013e3181ed61a3

Colley, R., Gorber, S. C., \& Tremblay, M. S. (2010). Quality control and data reduction procedures for accelerometry-derived measures of physical activity. Health, 21, 63-69.

Dishman, R. K., Washburn, R. A., \& Schoeller, D. A. (2001). Measurement of physical activity. Quest, 53, 295-309. doi: 10.1080/00336297.2001.10491746

Esliger, D. W., Copeland, J. L., Barnes, J. D., \& Tremblay, M. S. (2005). Standardizing and optimizing the use of accelerometer data for free-living physical activity monitoring. Journal of Physical Activity and Health, 3, 366-383.

Evenson, K. R., \& Terry, J. W. (2009). Assessment of differing definitions of accelerometer nonwear time. Reserach Quarterly for Exercise and Sport, 80, 355-362.

Freedson, P. S., Melanson, E., \& Sirard, J. (1998). Calibration of the Computer Science and Applications, Inc. accelerometer. Medicine \& Science in Sports \& Exercise, 30, 777-781. doi: 10.1097/00005768-199805000-00021

Masse, L. C., Fuemmeler, B. F., Anderson, C. B., Matthews, C. E., Trost, S. G., Catellier, D. J., \& Treuth, M. (2005). Accelerometer data reduction: A comparison of four reduction algorithms on select outcome variables. Medicine \& Science in Sports \& Exercise, 37, 
S544-S554. doi: 10.1249/01.mss.0000185674.09066.8a

Matthews, C. E., Chen, K. Y., Freedson, P. S., Buchowski, M. S., Beech, B. M., Pate, R. R., \&Troiano, R. P. (2008). Amount of time spent in sedentary behaviors in the United States, 2003-2004. American Journal of Epidemiology, 167, 875-881. doi:

10.1093/aje/kwm390

McAuley, E., Wójcicki, T. R., White, S.M., Mailey, E. L., Szabo, A. N., Gothe, N., ...

Estabrooks, P. (2012). Physical activity, function, and quality of life: Design and methods of the FlexToBa ${ }^{\mathrm{TM}}$ trial. Contemporary Clinical Trials, 33, 228-236. doi:10.1016/j.cct.2011.10.002.

McAuley, E., Wojcicki, T.R., Gothe, N., Olson, E.A., Szabo, A. N., Fanning, J., ... Mullen, S. P. (2013). Effects of a DVD-delivered exercise intervention on physical function in older adults. Journals of Gerontology: Medical Sciences. doi:10.1093/gerona/glt014

Owen, N., Healy, G. N., Matthews, C. E., \& Dunstan, D. W. (2010). Too much sitting: The population health science of sedentary behavior. Exercise and Sport Sciences Reviews, 38, 105-113. doi: 10.1097/JES.0b013e3181e373a2

Riddoch, C., Andersen, L., Wedderkopp, N., Harro, M., Klasson-Heggebo, L., Sardinha, L., et al. (2004). Physical activity levels and patterns of 9- and 15-year-old European children. Medicine \& Science in Sports \& Exercise, 36, 86-92.

Rowlands, A. V., Esliger, D. W., Eady, J., \& Eston, R. G. (2010). Empirical evidence to inform decisions regarding identification of non-wear periods from accelerometer habitual physical activity data. In G. Baquet \& S. Berthoin (Eds.), Chidren and Exercise XXV: The proceedings of the $25^{\text {th }}$ pediatric work physiology meeting (pp. 219-222). New York: Routledge. 
Sasaki, J. E., John, D., \& Freedson, P. S. (2011). Validation and comparison of ActiGraph activity monitors. Journal of Science and Medicine in Sport, 14, 411-416. doi:10.1016/j.jsams.2011.04.003

Shephard, R. J. (2003). Limits to the measurement of habitual physical activity by questionnaires. British Journal of Sports Medicine, 37, 197-206. doi: 10.1136/bjsm.37.3.197

Troiano, R. P., Berrigan, D., Dodd, K. W., Mâsse, L. C., Tilert, T., McDowell, M. (2008). Physical activity in the United States measured by accelerometer. Medicine \& Science in Sports \& Exercise, 40, 181-188. doi: 10.1249/mss.0b013e31815a5lb3

Ward, D. S., Evenson, K. R., Vaughn, A., Brown Rodgers, A., \& Troiano, R. P. (2005). Accelerometer use in physical activity: Best practices and research recommendations. Medicine \& Science in Sports \& Exercise, 37, S582-S588. doi: 10.1249/01.mss.0000185292.71933.91

Winkler, E. A. H., Gardiner, P. A., Clark, B. K., Matthews, C. E., Owen, N., \& Healy, G. N. (2012). Identifying sedentary time using automated estimates of accelerometer wear time. British Journal of Sports Medicine, 46, 436-442. doi: 10.1136/bjsm.2010.079699 
Table 1. Percentage of participants with 1-7 valid days for 20, 30, and 60 minute interruption periods, and based on self-reported wear time

\begin{tabular}{cccccccc}
\hline $\begin{array}{c}\text { Interruption } \\
\text { period }\end{array}$ & $\begin{array}{c}\mathbf{1} \text { valid } \\
\text { day }\end{array}$ & $\begin{array}{c}\mathbf{2} \text { valid } \\
\text { days }\end{array}$ & $\begin{array}{c}\mathbf{3} \text { valid } \\
\text { days }\end{array}$ & $\begin{array}{c}\mathbf{4} \text { valid } \\
\text { days }\end{array}$ & $\begin{array}{c}\mathbf{5} \text { valid } \\
\text { days }\end{array}$ & $\begin{array}{c}\mathbf{6} \text { valid } \\
\text { days }\end{array}$ & $\begin{array}{c}\mathbf{7} \text { valid } \\
\text { days }\end{array}$ \\
\hline 20 & 92.6 & 85.5 & 80.4 & 71.7 & 59.2 & 41.5 & 24.8 \\
30 & 99.7 & 98.4 & 97.1 & 95.5 & 89.4 & 78.5 & 59.5 \\
60 & 100 & 99.7 & 99.0 & 98.7 & 97.7 & 95.8 & 84.9 \\
Self-report & 99.9 & 99.6 & 98.9 & 98.6 & 97.6 & 94.6 & 77.2 \\
\hline
\end{tabular}


Figure 1. Mean wear time based on self-report, 20, 30, and 60-minute interruption periods

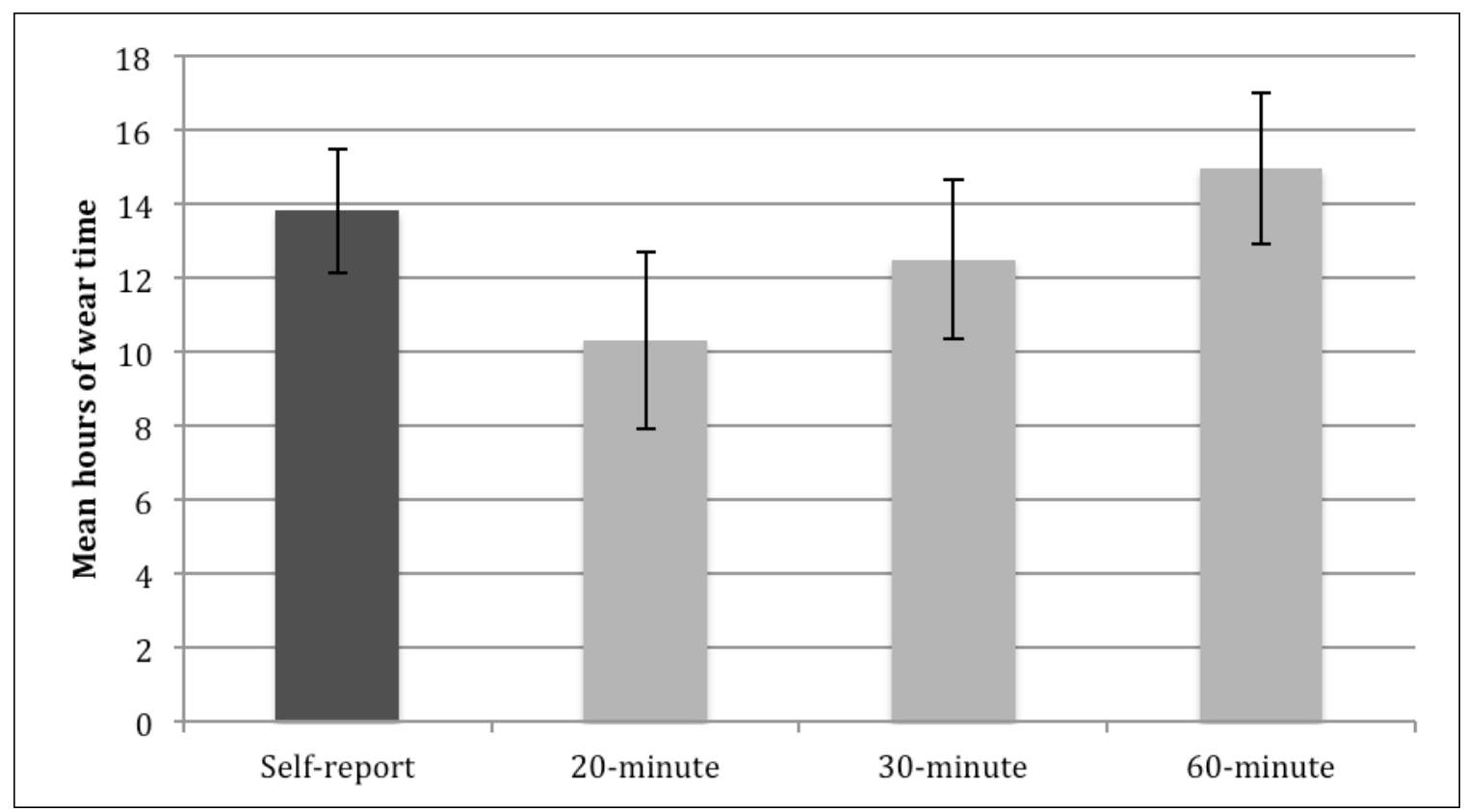


Figure 2. Percentage of participants with 1-7 valid days for 20, 30, and 60 minute interruption periods, and based on self-reported wear time

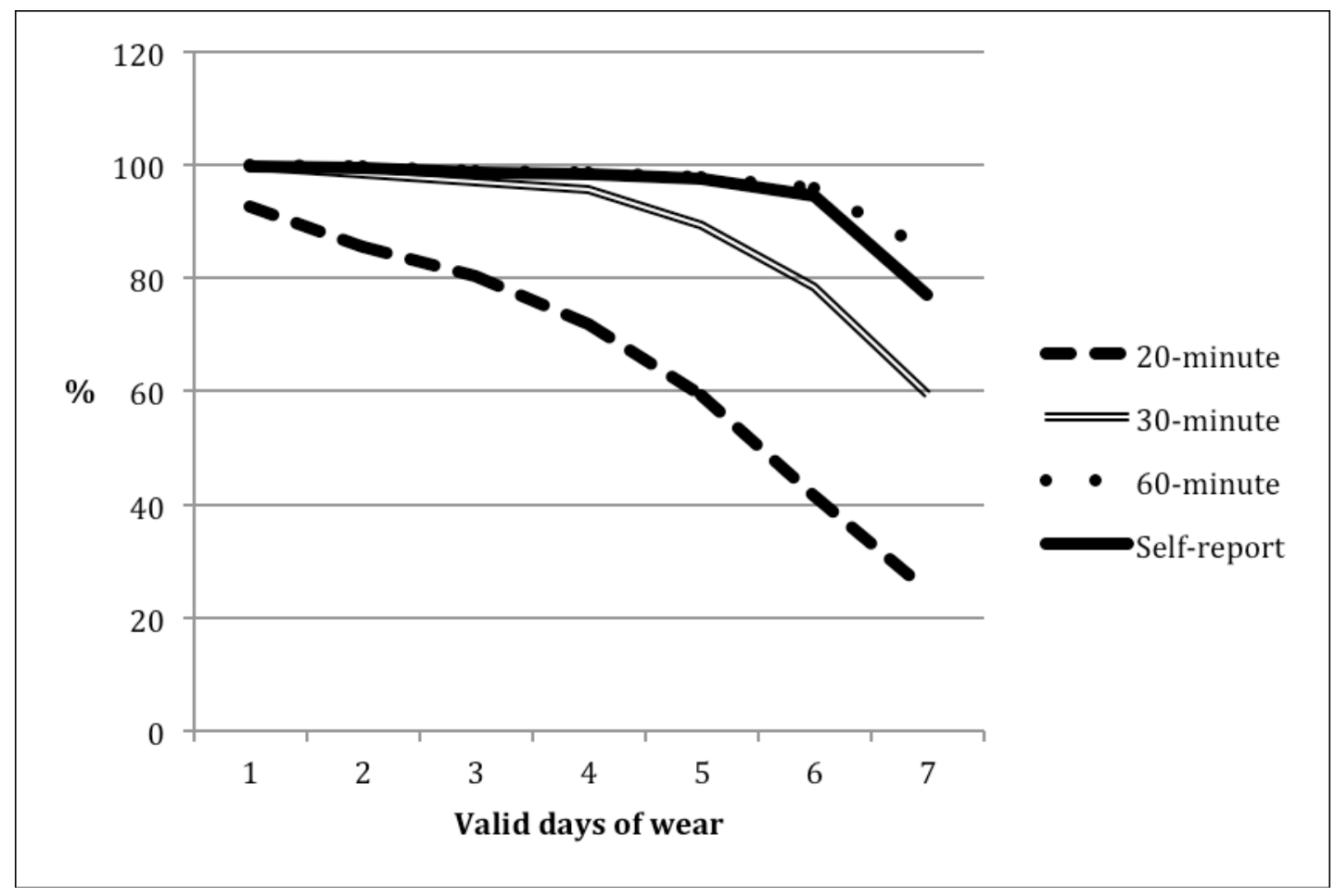

